\title{
Etude Ethnobotanique Sur L'utilisation De Mentha Pulegium, Mentha Piperita Et Pelargonium Graveolens Au Nord Du Maroc (Taounate) Et Évaluation De Leur Pouvoir Antimicrobien
}

\begin{abstract}
M. Chraibi
K. Fikri-Benbrahim

Laboratoire de Biotechnologie Microbienne, Faculté des Sciences et Techniques, Université Sidi Mohamed Ben Abdellah (USMBA)-Fès, Maroc M. Amrani

Laboratoire de Physiologie, Pharmacologie et Santé environnementale, Faculté des Sciences, USMBA, Fès, Maroc

\section{A. Farah}

Laboratoire de Chimie Organique, Faculté des Sciences et Techniques, USMBA-Fès, Maroc

\section{A. Bari}

Laboratoire de Biotechnologie et Préservation des Ressources Naturelles, Faculté des Sciences, USMBA, Fès, Maroc

\section{Z. Benziane Ouaritini}

Laboratoire de Physiologie, Pharmacologie et Santé environnementale, Faculté des Sciences, USMBA, Fès, Maroc

Doi: 10.19044/esj.2018.v14n24p113 URL:http://dx.doi.org/10.19044/esj.2018.v14n24p113

Abstract

Traditional medicine has always occupied an important place in Moroccan traditions medication, Taounate region is concrete example. Thus, this survey aims to highlight the different uses of Mentha pulegium, Mentha piperita and Pelargonium graveolens in Taounate city during April month 2017, and to evaluate their antimicrobial potency against Staphylococcus epidermidis and Acinetobacter baumannii by microdilution method. A questionnaire was used with residents, herbalists and phytotherapists. The results showed that these three plants are used mainly for their therapeutic virtues; precisely against digestive disorders representing the most cited diseases for Mentha piperita (51\%), respiratory diseases for Mentha pulegium $(50 \%)$ and dermatological conditions for Pelargonium graveolens $(44 \%)$. The majority of remedies are prepared by decoction (56\%) from leaves (71\%) and usually orally administered $(68 \%)$ as herbal tea $(58 \%)$. Cure rate is $41 \%$ with
\end{abstract}


$53 \%$ health improvement. In addition, the essential oils of these studied plants exhibited strong inhibitory effect against all tested microorganisms with minimum inhibitory concentrations ranging from $0.01562 \%$ to $1 \%(\mathrm{v} / \mathrm{v})$.

Keywords: Traditional medicine, Taounate region, Mentha pulegium, Mentha piperita, Pelargonium graveolens, Taounate, ethnobotanical survey, therapeutic uses, antimicrobial potency.

\section{Resume}

La médecine traditionnelle a toujours occupé une place importante dans les traditions de médication au Maroc. La région de Taounate en est un exemple concret. Ainsi, une enquête a été effectuée dans cette zone ayant pour objectif de mettre en évidence les différentes utilisations de Mentha pulegium, Mentha piperita et Pelargonium graveolens dans la ville de Taounate durant le mois d'Avril 2017 et d'évaluer leur pouvoir antimicrobien contre Staphylococcus epidermis et Acinetobacter baumannii par la méthode de microdilution. Pour cela, un questionnaire a été utilisé auprès des habitants, des herboristes et des phytothérapeutes. Les résultats ont montré que ces trois plantes sont utilisées principalement pour leurs vertus thérapeutiques, précisément contre les affections digestives représentant les maladies les plus citées pour Mentha piperita (51\%), les affections respiratoires pour Mentha pulegium (50\%) et les affections dermatologiques pour Pelargonium graveolens (44\%). La majorité des remèdes sont préparées par décoction $(56 \%)$ à partir des feuilles $(71 \%)$ et généralement administrées par voie orale (68\%) sous forme de tisane (58\%). Le taux de guérison est de $41 \%$ avec 53\% d'amélioration. Par ailleurs, les huiles essentielles de ces trois plantes ont présenté un fort pouvoir inhibiteur contre les souches testées avec des concentrations minimales inhibitrices qui varient de $0,01562 \%$ à $1 \%$ (v/v).

Mots clés : Médecine traditionnelle, région de Taounate, Mentha pulegium, Mentha piperita, pelargonium graveolens, enquête ethnobotanique, utilisations thérapeutiques, pouvoir antimicrobien.

\section{Introduction}

Pendant la dernière décennie, le recours à la médecine traditionnelle a connu un regain d'attention et d'intérêt dans le monde (Franchomme et al., 1990). Grâce aux contrastes géographiques, le Maroc offre une gamme variée de bioclimats méditerranéens permettant le développement d'une flore riche et diversifiée avec un taux élevé d'endémisme constituée de plus de 4200 espèces, ce qui le rend un fournisseur traditionnel du marché mondial en plantes aromatiques et médicinales (El Rhaffari et Zaid, 2002). De plus, la population marocaine dispose d'un savoir-faire ancestral dans la médication 
par les plantes grâce à sa tradition riche dans le domaine de la phytothérapie. Il s'agit d'un héritage arabo-berbère, largement influencé par la religion islamique; ainsi, l'utilisation d'un certain nombre de plantes médicinales, dans le traitement de plusieurs maladies, est-elle une partie intégrée de la culture marocaine (Lamendin, 2004). Vu leurs utilisations dans divers domaines (pharmaceutique, cosmétique et industriel) ainsi que la découverte de leurs atouts, les plantes aromatiques et médicinales connaissent une demande de plus en plus forte sur les marchés mondiaux (Lorrain, 2013).

Connue localement sous le nom de "Fliou", Mentha pulegium L. pousse dans les zones humides des plaines et des montagnes jusqu'à 2200 mètres d'altitude. Au Maroc, elle est très répandue notamment entre les régions de Marrakech et d'Azrou. Appartenant à la famille des Lamiaceae, $M$. pulegium est une plante herbacée possédant des propriétés antibactérienne (Chraibi et al., 2017), antifongique (Benabed et al., 2017), antioxydante, antileishmanienne (Bouyahya et al., 2017), antiviral (Parsania et al., 2017), antiinflammatoire (Kogiannou et al., 2013), insecticide (Barros et al., 2015) et antimycobactérienne (Chraibi et al., 2016).

Mentha piperita ou menthe poivrée est issue de l'hybride naturel de Mentha aquatiqua L. (menthe aquatique) et Mentha spicata L. (menthe verte). Bien qu'étant une espèce indigène de la région méditerranéenne, elle est cultivée partout dans le monde. Cette plante a été rapportée par d'autres travaux pour son pouvoir insecticide (Boualem et al., 2017), antimicrobien (Desam et al., 2017), antioxydant (Li et al., 2017) et anti-inflammatoire (Mogosan et al., 2017).

Pelargonium graveolens (Geraniaceae), appartient à la catégorie des plantes vivaces à feuillage odorant. Originaire d'Afrique du Sud, le Géranium rosat est cultivé dans de nombreuses régions méditerranéennes et subtropicales, et sa localisation détermine fortement sa composition biochimique. La bio-activité de cette plante a été rapportée dans plusieurs travaux (Hamidpour et al., 2017 ; Radan, 2017 ; Labarde, 2017 ; Chraibi et al., 2017).

C'est dans ce cadre que s'inscrit la présente étude qui a pour objectif de recueillir l'ensemble des informations sur les applications thérapeutiques et traditionnelles de Mentha pulegium, Mentha piperita et Pelargonium graveolens, dans la ville de Taounate. Ainsi que de déterminer la composition chimique de leurs huiles essentielles et d'évaluer leur pouvoir antimicrobien.

\section{Materiel Et Methode Zone d'étude}

Taounate est une subdivision à dominance rurale de la région marocaine de Fès-Meknès. Cette province, située dans le Sud du Rif et faisant partie du pays de Jebala, a des frontières avec la province de Chefchaouen au 
Nord, de Taza à l'Est, Ouezzane au Nord-Ouest, de Sidi Kacem à l'Ouest, et la préfecture de Fès au Sud.

\section{Méthodologie}

\section{Enquêtes ethnobotaniques}

L'enquête ethnobotanique est basée sur une série de collectes réalisées en utilisant un questionnaire préétablie (Annexe I) soumis à une centaine de personnes, au cours d'un entretien individuel, d'une durée d'environ 30 minute chacun, qui s'est déroulé pendant un mois (Avril 2017). Durant chaque entretien, les informations sur les personnes questionnées (Age, niveau scolaire, situation familiale et profession) sur les plantes médicinales étudiées (Caractères et usages) sont collectées.

\section{Traitement des données}

Les données inscrites sur des fiches brutes ont été transférées dans une base de données et traitées par le logiciel de traitement statistique IBM SPSS version 21 .

\section{Extraction des huiles essentielles}

Les parties aériennes de chacune des trois plantes étudiées ont été soumises à une hydrodistillation pendant $3 \mathrm{~h}$ en utilisant un appareil de type Clevenger. Chaque huile essentielle recueillie a été maintenue dans l'obscurité à $4{ }^{\circ} \mathrm{C}$ jusqu'à utilisation ultérieure.

\section{Analyse de la composition chimique des huiles essentielles}

Les huiles essentielles ont été analysées en utilisant la Chromatographie en phase gazeuse-couplée à la spectrométrie de masse (CPG-SM) (Polaris Q pour piéger les ions SM). Ainsi, les analyses ont été effectuées sur un chromatographe en phase gazeuse Hewlett-Packard (HP 6890) (détecteur à ionisation de flamme), équipé d'une colonne capillaire 5\% phénylméthyl silicone HP-5 (30 m x 0,25 mm x épaisseur 0,25 $\mu \mathrm{m})$. La température a été programmée à partir de $50{ }^{\circ} \mathrm{C}$, après 5 min de maintien initial, à $200{ }^{\circ} \mathrm{C}$ à $4{ }^{\circ} \mathrm{C} / \mathrm{min}$. Le gaz vecteur de Chromatographie était $\mathrm{N}_{2}(1,8$ $\mathrm{ml} / \mathrm{min}$ ); le mode partagé a été utilisé avec un débit de 72,1 $\mathrm{ml} / \mathrm{min}$ et un rapport de 1/50; la température de l'injecteur et du détecteur était de $250{ }^{\circ} \mathrm{C}$, et le temps de maintien final était de 48 minutes. La machine était dirigée par un système informatique de type "HP Chem Station", gérant son fonctionnement et permettant de suivre l'évolution des analyses chromatographiques. Les échantillons dilués (1/20 dans du méthanol) ont été injectés manuellement à raison de $1 \mu \mathrm{L}$. 


\section{Détermination de la concentration minimale inhibitrice (CMI) contre les souches testées}

La concentration minimale inhibitrice (CMI) est définie comme la plus faible concentration en huile essentielle capable d'inhiber toute croissance bactérienne. Elle a été déterminée grâce à l'utilisation des microplaques à 96 puits avec une gamme de concentrations des huiles essentielles allant de 4 à $0,0078 \%$. Les microplaques ont été incubées à $37^{\circ} \mathrm{C}$ pendant 24 heures. Ensuite, $10 \mu \mathrm{l}$ de résazurine ont été ajoutés pour révéler la croissance bactérienne (Chraibi et al., 2017). Après une incubation à $37^{\circ} \mathrm{C}$ pendant 2 heures, les CMI ont été déterminées comme les plus faibles concentrations en huiles essentielles ayant montré un changement de coloration de la résazurine. La croissance bactérienne est détectée par la réduction de la résazurine ayant une coloration bleue en résorufine ayant une coloration rose.

\section{Resultats Et Discussion}

L'étude ethnobotanique a permis de recueillir plusieurs informations sur l'enquêté et sur l'usage de la plante.

\section{Profil des informateurs}

\section{Age}

L'utilisation des plantes étudiées est répandue chez l'ensemble des personnes questionnées, avec une prédominance chez les personnes âgées de 40 à 50 ans (25\%). Cependant, un taux de $22 \%$ est noté pour la tranche d'âge de 30 à 40 ans. L'utilisation est moins importante (3\%) chez les informateurs les moins âgés (<20) (Fig. 1).

Le pourcentage élevé des utilisateurs âgés de 30 à 50 ans montre que ces personnes ont tendance à utiliser ces plantes de façon importante. Ces résultats indiquent qu'au niveau de la région de Taounate, la connaissance des propriétés et des usages de la plante étudiée dépend de la longue expérience, et de la confiance que ces personnes éprouvent pour la médecine traditionnelle ; ceci est en accord avec d'autres travaux faits dans la région de Mechraâ Bel Ksiri, Région du Gharb du Maroc. (Benkhnigue et al., 2011).

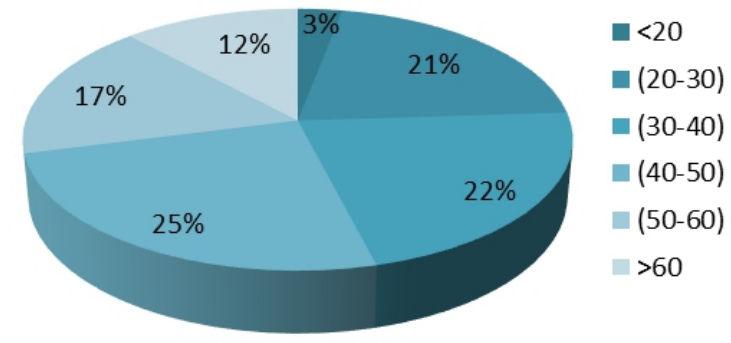

Fig. 1 Répartition des utilisateurs des plantes étudiées selon la tranche d'âge 


\section{Genre}

L'utilisation des plantes étudiées dans la région de Taounate ne dépend pas du sexe, puisque $44 \%$ des utilisateurs sont des hommes et $56 \%$ sont des femmes (Fig. 2).

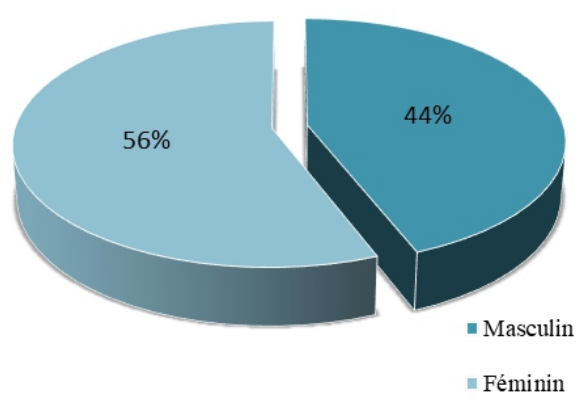

Fig. 2 Répartition des utilisateurs des plantes étudiées selon le genre

\section{Situation familiale}

Les plantes étudiées sont beaucoup plus utilisées par les personnes mariées (67\%) que par les célibataires (33\%) (Fig. 3). Ces résultats concordent avec ceux obtenus par d'autres auteurs (Benkhnigue et al., 2011 ; Hafsé et al., 2015). Ceci peut s'expliquer par le fait que cette utilisation permet aux personnes mariées de minimiser les charges matérielles dépensées pour l'achat des médicaments synthétiques.

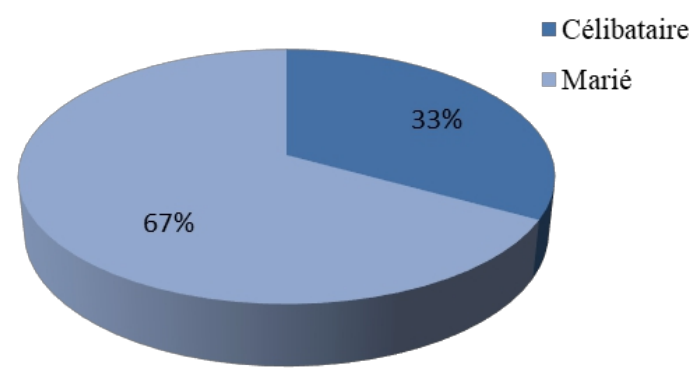

Fig. 3 Répartition des utilisateurs des plantes étudiés selon la situation familiale

\section{Niveau d'étude}

Dans la zone d'étude, $32 \%$ des utilisateurs des plantes étudiées sont analphabètes, alors que les $68 \%$ correspondent à différents niveaux intellectuels (20\% primaire, $25 \%$ secondaire et $23 \%$ universitaire) (Fig. 4). 

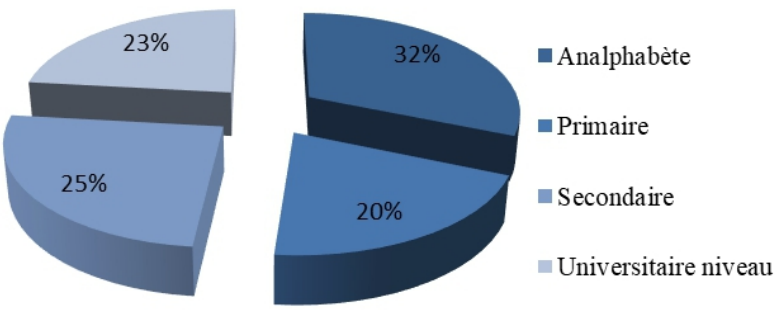

Fig. 4 Répartition des utilisateurs des plantes étudiés selon le niveau d'étude

\section{Niveau socio-économique (NSE)}

Dans notre étude, $59 \%$ de la population faisant usage des plantes étudiées ont un Niveau socio-économique (NSE) moyen, 32\% appartiennent à un niveau bas, et seulement $9 \%$ disposent d'un NSE élevé (Fig. 5).

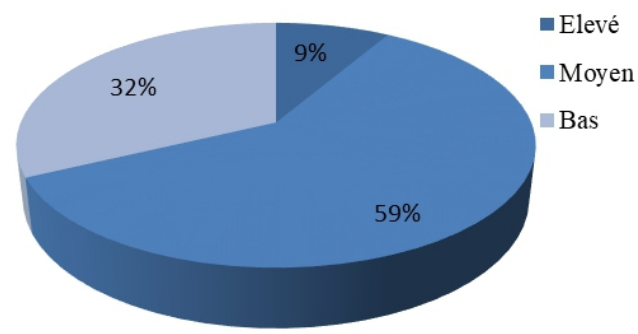

Fig. 5 Répartition des utilisateurs des plantes étudiés selon le NSE

\section{Profession}

La figure 6 montre que $41 \%$ des informateurs sont au chômage, alors que $6 \%$ et $16 \%$ des personnes interrogées représentent respectivement les phytothérapeutes et les herboristes. Les $37 \%$ restants sont des agriculteurs, des fonctionnaires et des commerçants (Fig. 6). Ceci reflète l'image du transfert des pratiques traditionnelles d'une génération à l'autre (Benkhnigue et al., 2011). Malheureusement, la transmission de cette connaissance est actuellement en danger parce qu'elle n'est pas toujours assurée (Anyinam, 1995).

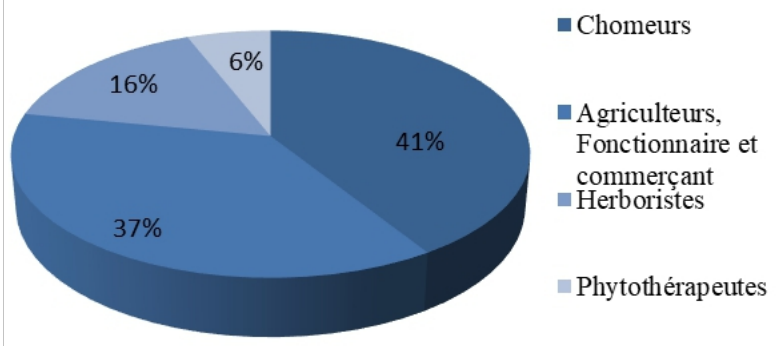

Fig. 6 Répartition des utilisateurs des plantes étudiées selon leur profession 


\section{Données sur le type de médecine utilisée par la population}

Les informations recueillies au niveau de la région de Taounate indiquent un pourcentage important des usagers de la médecine traditionnelle. En effet, $77 \%$ de la population de cette zone s'adressent à la médecine traditionnelle, par contre seuls $23 \%$ ont recours à la médecine moderne (Figure 7). Parmi ceux qui ont recours à la médecine traditionnelle, $51 \%$ l'ont choisi pour son efficacité, $32 \%$ : pensent que c'est moins cher, $13 \%$ : pour des raisons d'acquisition, et seuls $4 \%$ pensent que les médicaments sont inefficaces.

Alors que parmi ceux qui ont recours à la médecine moderne, $62 \%$ des informateurs pensent qu'elle est plus précise que la médecine traditionnelle, $33 \%$ s'adressent à cette médecine parce qu'elle est efficace et $5 \%$ à cause de la possibilité de la toxicité des plantes.

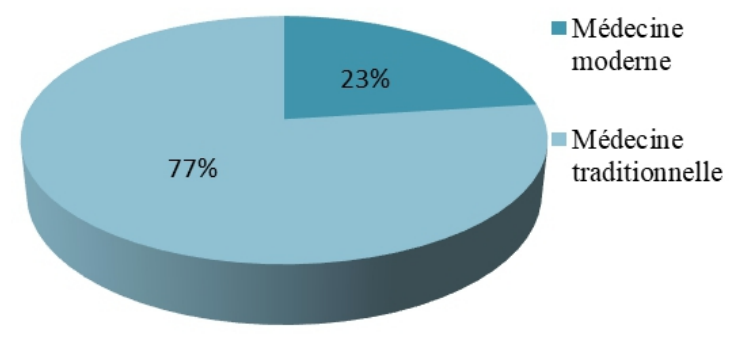

Fig. 7 Données sur le type de médecine utilisée par la population

\section{Données sur l'utilisation des plantes médicinales selon le degré de possession des informations}

$43 \%$ de la population se réfèrent aux expériences des autres, $36 \%$ aux herboristes, $19 \%$ des personnes se réfèrent à elles-mêmes soit en consultant les livres de la médecine traditionnelle soit en suivant les programmes télévisés ou bien en se basant sur leur propre expérience grâce à l'existence de nombreuses plantes médicinales dans leur entourage, et $2 \%$ seulement consultent les pharmaciens (Figure 8).

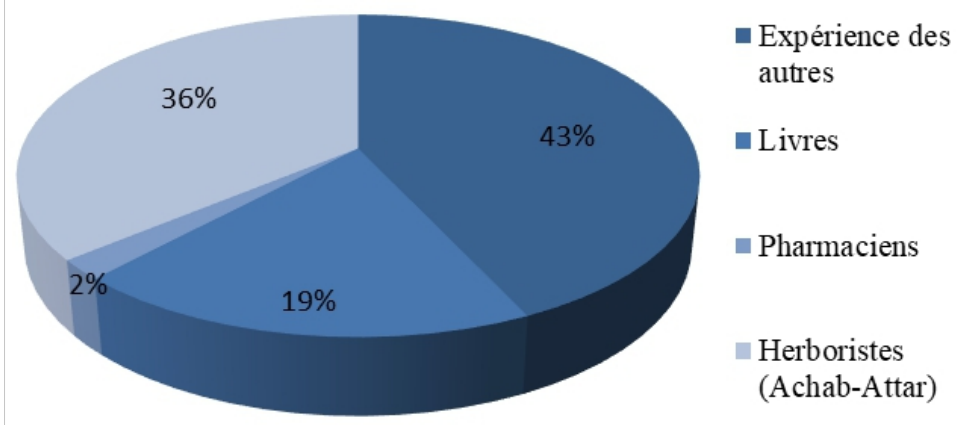

Fig. 8 Répartition des utilisateurs des plantes médicinales selon le mode de possession des informations concernant l'utilisation des plantes médicinales 


\section{Efficacité des plantes}

$39 \%$ des gens de la région de Taounate estiment que les plantes médicinales permettent une guérison des maladies traitées ; $54 \%$ trouvent qu'elles permettent seulement une amélioration de l'état de la santé; $4 \%$ croient que les plantes médicinales provoquent une évolution de la maladie, alors que 3\% pensent qu'elles provoquent des effets secondaires (Fig. 9).

Presque la totalité des personnes enquêtées diagnostiquent ellesmêmes leurs maux (91\%). Elles sont satisfaites des résultats, car $40 \%$ estiment que les plantes étudiées permettent une guérison des maladies traitées et 53\% d'entre elles pensent que les plantes utilisées contribuent à une amélioration de leur état de santé, alors que $7 \%$ de la population locale croient que les plantes étudiés ont des effets secondaires ; des états de toxicité et même une aggravation des maladies (Fig. 10).

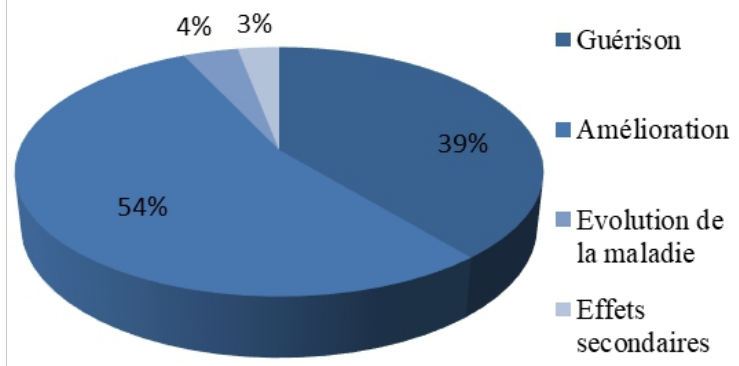

Fig. 9 Résultats des soins par les plantes
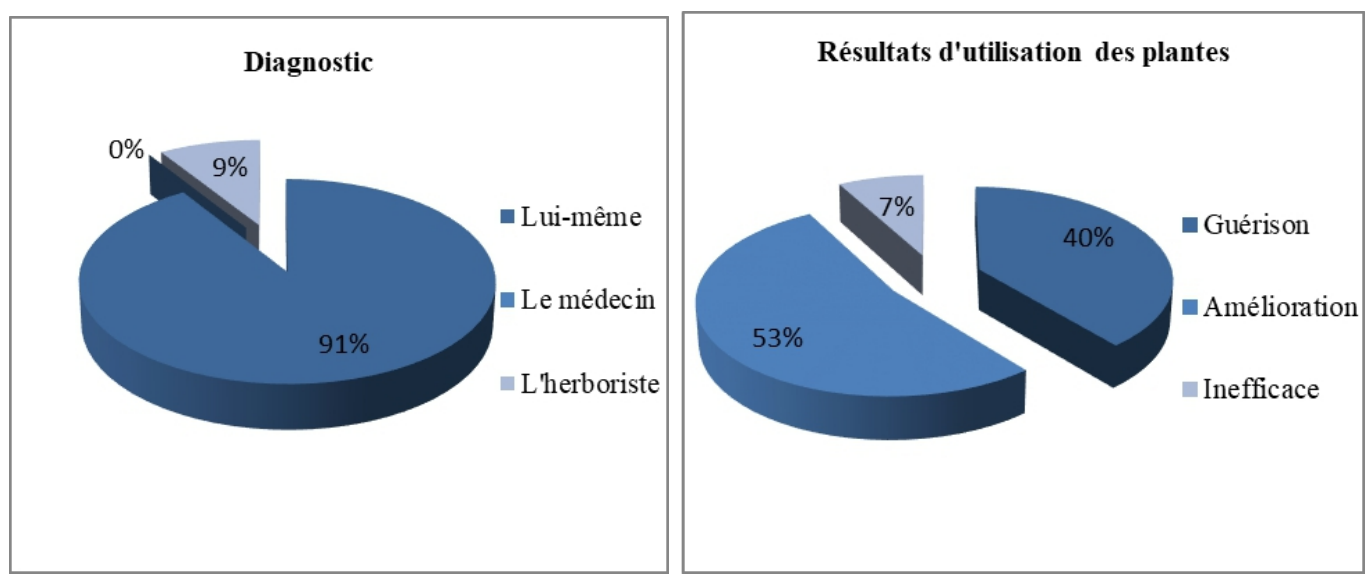

Fig. 10 Diagnostic et résultats des soins par les plantes

\section{Nature d'usage}

Les informations recueillies sur les utilisations thérapeutiques et traditionnelles locales de Mentha pulegium ont révélé qu'un taux de $81 \%$ d'usage est thérapeutique; $9 \%$ correspond à l'usage thérapeutique et 
cosmétique, 7\% à l'usage cosmétique et 3\% aux autres utilisations (Figure 11a).

Pour $P$. graveolens $49 \%$ d'usage est thérapeutique; $36 \%$ correspond à l'usage thérapeutique et cosmétique, $9 \%$ à l'usage cosmétique et $6 \%$ aux autres utilisations (Figure 11-b).

Pour Mentha piperita, 58\% d'usage est thérapeutique; $28 \%$ correspond à l'usage thérapeutique et cosmétique, $9 \%$ à l'usage cosmétique et $5 \%$ aux autres utilisations (Figure 11-b).

\section{(a) Mentha pulegium}

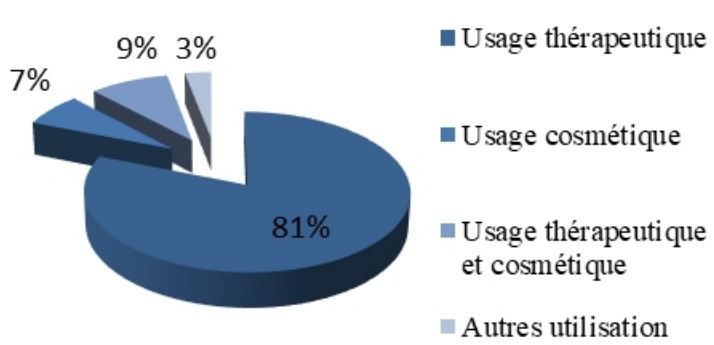

\section{(b) Pelargonium graveolens}

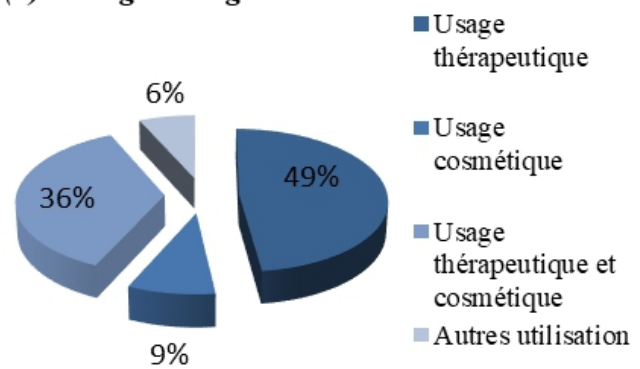

(c) Mentha piperita

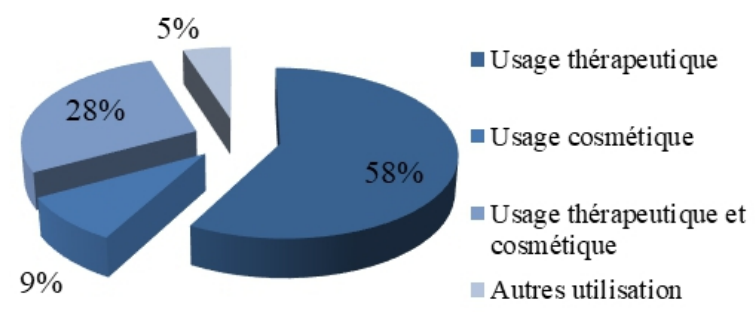

Fig. 11 Répartition de l'utilisation des plantes étudiées selon la nature d'usage

\section{Les parties utilisées}

Dans la zone d'étude, et pour les trois plantes, les feuilles sont les parties les plus utilisées avec un pourcentage de $71 \%$; suivies par la combinaison des feuilles et tiges (15\%), puis la combinaison des feuilles, tiges et fleurs (7\%) et la plante entière (7\%) (Fig. 12).

Ce résultat peut être expliqué par le fait que ces feuilles sont à la fois le centre des réactions photochimiques et le réservoir de matières organiques qui en dérivent. Elles fournissent la majorité des alcaloïdes, hétérosides et huiles essentielles. En effet, il a été rapporté qu'elles sont pourvues d'activités 
anti-inflammatoire, antibactérienne, antifongique, antipyrétique, astringente etc. (Benkhnigue et al., 2011 ; Sachin et al., 2012 ; Saraswathi et al., 2011).

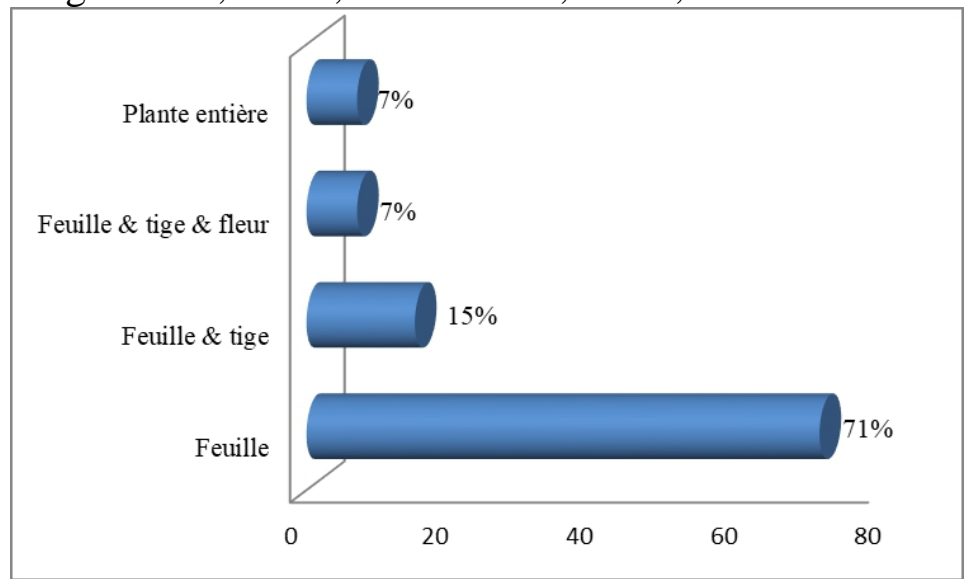

Fig. 12 Répartition de l'utilisation des plantes étudiées selon leurs parties utilisées

\section{Techniques de récolte}

$96 \%$ de la population ont recours à des techniques manuelles lors de la récolte des plantes alors que seuls $4 \%$ utilisent des techniques mécaniques (Fig. 13).

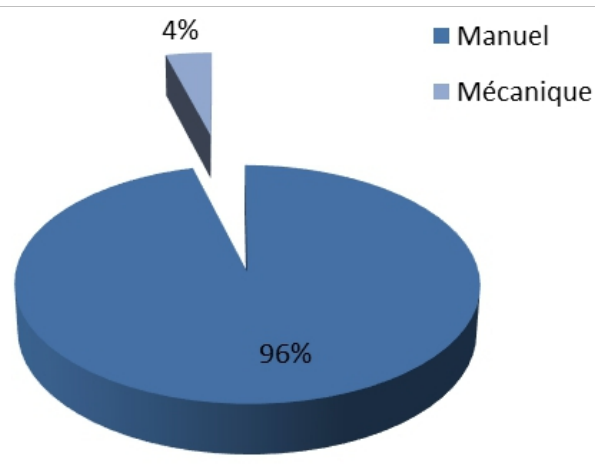

Fig. 13 Technique de récolte

\section{Méthodes de séchage et modes de conservation}

Le séchage et la conservation se font à l'abri de la lumière; ce qui permet la préservation de la majorité des principes actifs des plantes (Fig. 14). 


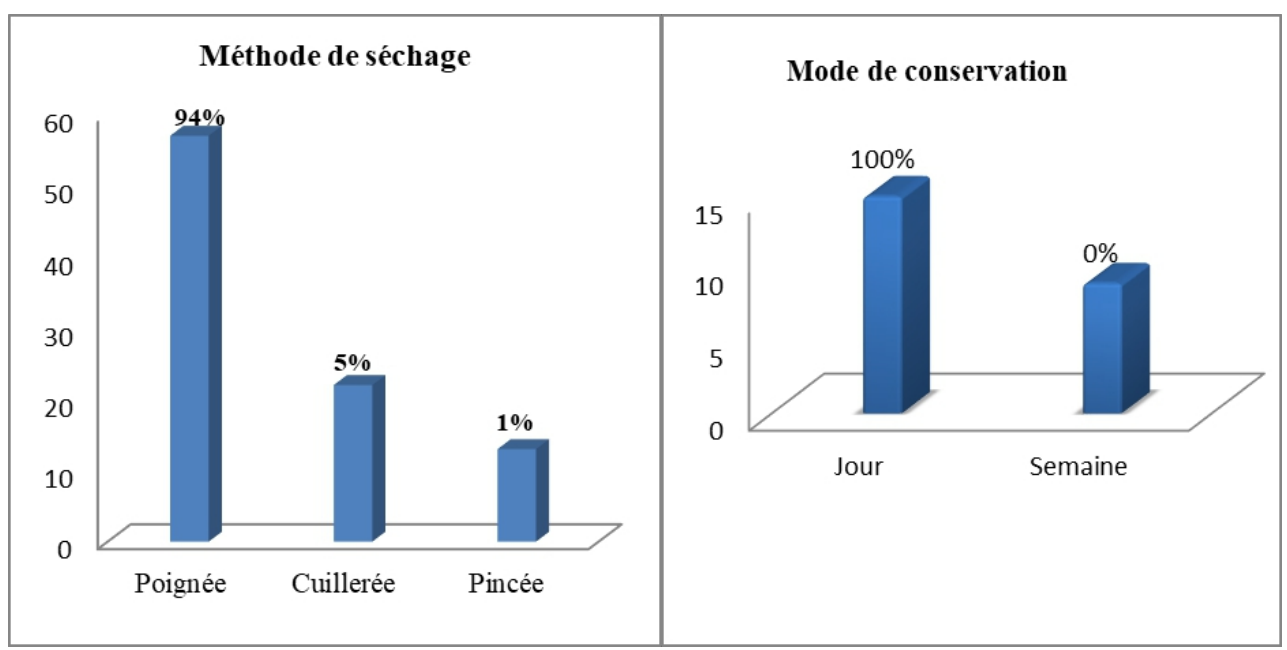

Fig. 14 Méthode de séchage et mode de conservation

Forme d'emploi, dose utilisée, mode de préparation et d'administration

Les plantes étudiées sont utilisées sous différentes formes; tisane (58\%), Poudre (23\%), combinaison de tisane et de poudre (12\%) et huile essentielle (7\%) (Fig. 15).

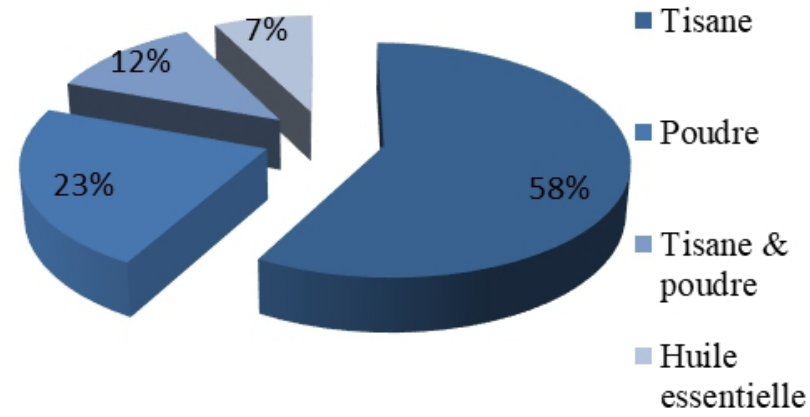

Fig. 15 Répartition des utilisations des plantes étudiées selon la forme d'emploi

\section{Dose utilisée et durée d'utilisation}

92\% des utilisateurs des plantes étudiées dans le cercle de Taounate utilisent les plantes avec des doses non précises, dont $13 \%$ par pincée, $22 \%$ par cuillerée et $57 \%$ par poignée.

La dose reste encore aléatoire; ce qui se manifeste par des effets néfastes sur la santé «aucune substance n'est poison elle-même, c'est la dose qui fait le poison» (Benkhnigue et al., 2011). Alors que $8 \%$ de la population utilisent les plantes médicinales avec des doses bien précises.

La poignée est la dose la plus utilisée (57\%) et dans la quasi-totalité des cas, cette dose est administrée jusqu'à guérison. Néanmoins, cette absence de notion de posologie exacte chez la population locale peut se manifester par 
des effets néfastes sur la santé car il y a souvent une toxicité dose-dépendante (Fig.16).

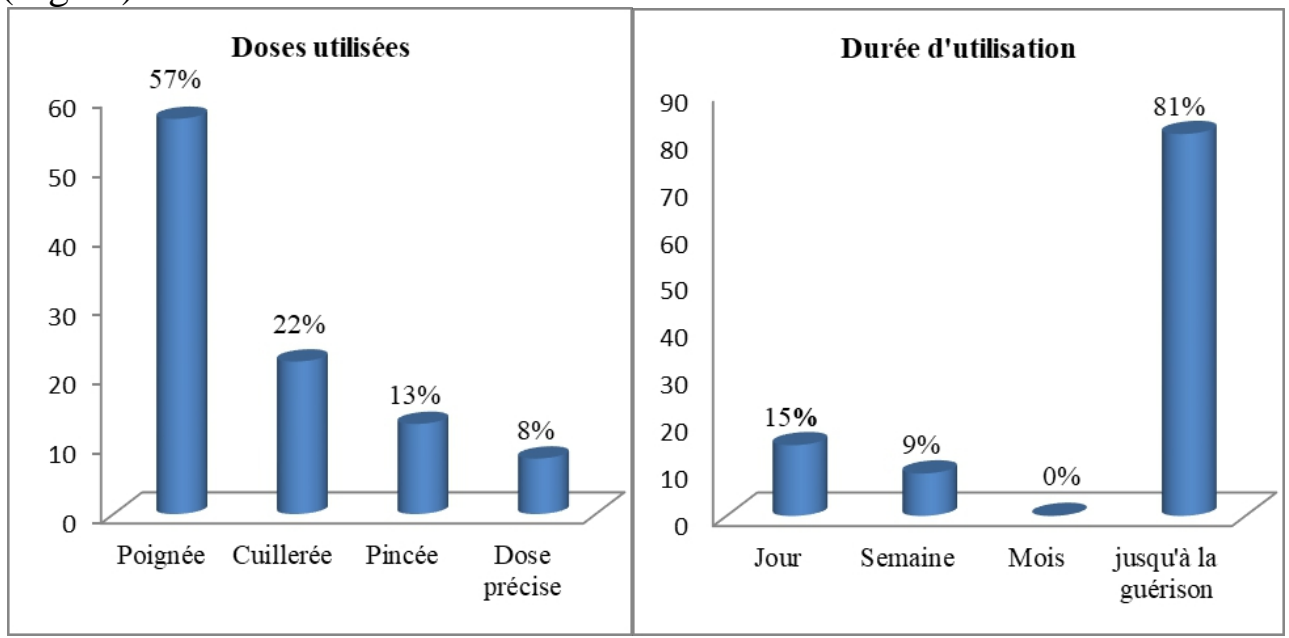

Fig. 16 Répartition des utilisateurs des plantes étudiées selon la posologie et la durée d'utilisation

Afin d'obtenir des dérivés de la plante étudiée, plusieurs modes de préparations sont employés à savoir la décoction, l'infusion et le cataplasme. La décoction constitue le mode de préparation le plus fréquent $(56 \%)$ alors que les autres techniques de préparations (cataplasme, infusion, décoction et autres techniques) représentent 44\% (Fig. 17 a).

Concernant le mode d'administration, $68 \%$ des dérivés de la plante (tisane, poudre etc.) sont administrés par voie orale, $6 \%$ par massage et correspondent aux huiles essentielles, les $36 \%$ restant rassemblent les autres modes d'administrations (Fig. 17 b).

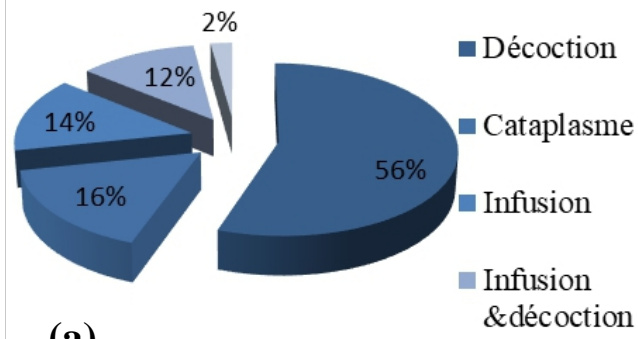

(a)

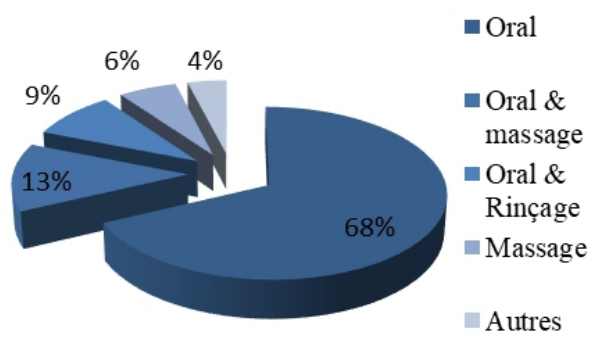

(b)

Fig. 17 Répartition des utilisateurs des plantes étudiés selon le mode de préparation (a) et selon le mode d'administration (b)

\section{Maladies traitées}

L'enquête ethnobotanique a révélé que Mentha pulegium est consommée principalement pour le traitement des maladies respiratoires 
(50\%). Elle est également utilisée contre les affections du tube digestif (16\%). Le pourcentage d'utilisation de la plante pour le traitement des affections ostéo-articulaires et neurologiques est de l'ordre de $15 \%$ pour chacun et les autres utilisations représentent un pourcentage de 4\% (Tableau 1).

Mentha piperita est consommé principalement pour le traitement des maladies de l'appareil digestif (51\%). Elle est également utilisée contre les affections neurologiques (29\%). Le pourcentage d'utilisation de la plante pour le traitement des maladies de l'appareil respiratoire est de (13\%) et d'autres utilisations représentent un pourcentage de $7 \%$ (Tableau 1).

Par ailleurs, $P$. graveolens est consommé principalement pour le traitement des affections dermatologiques (44\%). Le pourcentage d'utilisation de cette plante contre les affections neurologiques est de $16 \%$; pour le traitement des maladies de l'appareil digestif $(14 \%)$, le traitement des affections ostéo-articulaires (12\%), le traitement des affections respiratoires (7\%) et cardio-vasculaire (3\%). Les autres utilisations représentent un pourcentage de $(4 \%)$. L'ensemble des informateurs ont indiqué l'absence totale d'effets secondaires et des intoxications suite à l'utilisation de cette plante (Tableau 1).

Tableau 1 : Pathologies traitées par les plantes étudiées dans la région de Taounate

Huile essentielle de la plante utilisée

\begin{tabular}{ccc} 
plante utilisée & Pathologies traitées & \% \\
\hline \multirow{3}{*}{ Mentha pulegium } & Affections respiratoires & 50 \\
& Affections du tube digestif & 16 \\
& Affections neurologiques & 15 \\
& Affections ostéo-articulaires & 15 \\
& Autres utilisations & 4 \\
\hline \multirow{3}{*}{ Mentha piperita } & Affections du tube digestif & 51 \\
& Affections neurologiques & 29 \\
& Affections respiratoires & 13 \\
& Autres utilisations & 7 \\
\hline \multirow{3}{*}{ Pelargonium graveolens } & Affections dermatologiques & 44 \\
& Affections neurologiques & 16 \\
& Affections du tube digestif & 14 \\
& Affections ostéo-articulaires & 12 \\
& Affections respiratoires & 7 \\
& Affections cardio-vasculaires & 3 \\
& Autres utilisations & 4 \\
\hline
\end{tabular}

\section{Composition chimique des huiles essentielles testées}

A partir de l'analyse chromatographique, 18 composés ont été identifiés dans l'huile essentielle de Mentha pulegium où le pulégone est le principal constituant avec un pourcentage de $71,48 \%$; suivi du carvone $(5,66 \%)$ et du dihydrocarbone $(4,64 \%)$ (Tableau 2$)$. Une composition 
chimique similaire a été signalée pour cette huile essentielle en Inde, au Portugal et en Turquie, en particulier en ce qui concerne le contenu de pulégone qui est indiqué comme étant le composant majoritaire, mais avec des proportions différentes (Sachin et al., 2012 ; Lakhdar et al., 2015 ; Agnihotri et al., 2005 ; Teixeira et al., 2012).

Concernant l'huile essentielle de Mentha piperita, 27 composés volatils ont été détectés avec un total de 99,51\% de cette huile dont les composés majoritaires sont : le menthol $(46,32 \%)$, le menthofurane $(13,18 \%)$, l'acétate de menthyle $(12,10 \%)$, le menthone $(7,42 \%)$ ainsi que le 1,8 -cineole $(6,06 \%)$ (Tableau 3). Par conséquent, il est clair que le menthol est le principal composant majeur de cette huile essentielle, ce qui est en accord avec plusieurs autres travaux (Bouari et al., 2014 ; Benchikha et al., 2008).

Soixante et un différents composés, ont été révélés par l'analyse phytochimique, représentant 99,96\% de l'huile essentielle de Pelargonium graveolens, dont les principaux constituants étaient citronellol (26,98\%), géraniol $(14,12 \%)$, isomenthone $(8,80 \%)$, linalol $(4,97 \%)$, géranylformiate $(4,07 \%)$, le guaï-6,9-diène $(4,24 \%)$ et le citronellylformiate $(3,1 \%)$ (Tableau 4). Ces résultats sont concordants avec plusieurs études antérieures qui ont montré que le citronellol était le composant majeur de l'huile essentielle de cette plante (Saraswathi et al., 2011 ; Boukhatem et al., 2013 ; Boukhris et al., 2013).

Tableau 2. Composition chimique de l'huile essentielle de Menthapulegium

\begin{tabular}{|c|c|c|}
\hline IR & Constituants & $\%$ \\
\hline 939 & $\alpha$-pinene & 0,52 \\
\hline 952 & $\begin{array}{l}\text { Cyclohexanone-3- } \\
\text { methyl }\end{array}$ & 0,26 \\
\hline 980 & $\beta$-pinene & 0,39 \\
\hline 993 & Myrcene & 0,16 \\
\hline 994 & Octanol-3 & 1,86 \\
\hline 1001 & $\Delta$-2-carene & 0,07 \\
\hline 1031 & Limonène & 1,88 \\
\hline 1072 & p-mentha-3,8-diene & 1,44 \\
\hline 1154 & Menthone & 0,19 \\
\hline 1168 & Pinocarvone & 1,27 \\
\hline 1173 & Menthol & 0,72 \\
\hline 1194 & Dihydrocarvone & 4,64 \\
\hline 1238 & R(+)-pulégone & 71,48 \\
\hline 1242 & Carvone & 5,66 \\
\hline 1252 & Peperitone & 1,13 \\
\hline 1419 & Caryophyllene & 0,33 \\
\hline 1630 & $\beta$-eudesmol & 0,28 \\
\hline 1649 & $\gamma$-eudesmol & 0,49 \\
\hline & Total & 92,77 \\
\hline
\end{tabular}


Tableau 3. La composition chimique de l'huile essentielle de M. Piperitta

\begin{tabular}{clc}
\hline IR & Constituants & \% \\
\hline 931 & $\alpha$-thujene & 0.31 \\
939 & $\alpha$-Pinene & 0.32 \\
967 & Verbenene & 0.02 \\
975 & Sabinene & 1.38 \\
979 & $\beta$-pinene & 0.53 \\
1005 & $\alpha$-Phellandrene & 0.01 \\
1026 & P-cymene & 0.03 \\
1031 & Limonene & 3.01 \\
1033 & 1.8-Cineole & 6.06 \\
1070 & cis- & 0.24 \\
1098 & Linalool & 0.05 \\
1123 & Chrysanthenone & 0.42 \\
1152 & Menthone & 7.42 \\
1164 & Menthofuran & 13.1 \\
1165 & Neomenthol & 4.79 \\
1171 & Menthol & 46.3 \\
1177 & terpinen-4-ol & 0.04 \\
1189 & $\alpha$-terpineol & 0.03 \\
1243 & Carvone & 1.02 \\
1273 & Neomenthylacetat & 0.43 \\
1295 & Menthyl acétate & 12.1 \\
1305 & Isomenthylacetate & 0.82 \\
1352 & $\alpha$-terpinylacetate & 0.03 \\
1388 & $\beta$-Bourbonene & 0.37 \\
1409 & $\alpha$-Gurjunene & 0.03 \\
1418 & $\beta$-Caryophyllene & 0.55 \\
1460 & $\alpha$-humulene & 0.01 \\
\cline { 2 - 3 } Total & $\mathbf{9 9 . 5}$ \\
\hline & &
\end{tabular}

Tableau 4. La composition chimique de l'huile essentielle du géranium $P$. graveolens

\begin{tabular}{|c|c|c|}
\hline IR & Constituants & $\%$ \\
\hline 931 & $\alpha$-Thujene & 0.50 \\
\hline 1074 & Cis-oxidelinalol & 0.54 \\
\hline 1097 & Linalol & 4.97 \\
\hline 1111 & Cis-oxide rose & 0.82 \\
\hline 1134 & Terpinol-1 & 0.95 \\
\hline 1143 & Cis-Sabinol & 0.59 \\
\hline 1164 & Isomenthone & 8.80 \\
\hline 1189 & $\alpha$-Terpineol & 0.70 \\
\hline 1228 & Citronellol & 26.98 \\
\hline 1240 & Carvone & 0.53 \\
\hline 1255 & Geraniol & 14.12 \\
\hline 1270 & Geraniale & 0.73 \\
\hline 1275 & Citronellyl formiate & 7.09 \\
\hline 1300 & Geranyl Formiate & 4.07 \\
\hline 1349 & $\alpha$-Terpinylacetate & 0.54 \\
\hline 1353 & Citronellylacetate & 1.24 \\
\hline 1384 & $\beta$ - Bourbonene & 0.57 \\
\hline 1419 & $\beta$-Caryophyllene & 0.76 \\
\hline 1443 & Guaï-6,9-diene & 4.27 \\
\hline 1474 & $\beta$-Thujaplicin & 1.09 \\
\hline 1484 & $\gamma$-Thujaplicin & 0.71 \\
\hline 1526 & $\Delta$-Cadinene & 0.51 \\
\hline 1538 & $\alpha$-Cadinene & 0.56 \\
\hline 1558 & 1-nor-epi-Bouronanone & 1.09 \\
\hline 1585 & Nerylisovalerate & 0.96 \\
\hline 1586 & Davanone & 1.95 \\
\hline \multirow[t]{2}{*}{1697} & (E)-Citronellyltiglate & 1.42 \\
\hline & Total & 99.96 \\
\hline
\end{tabular}

\section{Activité antimicrobienne}

Les résultats de l'évaluation de l'activité antibactérienne des trois huiles essentielles contre Staphylococcus epidermidis et Acinetobacter baumannii sont présentés dans le tableau 5. Comme on peut le remarquer, les essences testées ont montré un effet antibactérien important. En effet, les valeurs de CMI de l'huile essentielle de Mentha pulegium vont de 0.25 à $1 \%$ $(\mathrm{v} / \mathrm{v})$, alors qu'elles varient de 0.01562 à $0.03125 \%(\mathrm{v} / \mathrm{v})$ et de 0.0625 à $0.25 \%$ $(\mathrm{v} / \mathrm{v})$ respectivement pour l'huile essentielle de Mentha piperita et de Pelargonium graveolens contre les souches étudiées. 
Tableau 5: Détermination des valeurs de CMI des huiles essentielles de M. pulegium, $M$. piperita et $P$. graveolens contre Staphylococcus epidermis et Acinetobacter baumannii

\begin{tabular}{ccccccc}
\hline \multirow{2}{*}{$\begin{array}{c}\text { Concentrati } \\
\text { on \% }\end{array}$} & \multicolumn{2}{c}{ Mentha pulegium } & \multicolumn{2}{c}{ Mentha piperita } & \multicolumn{2}{c}{ Pelargonium graveolens } \\
\cline { 2 - 7 } & $\begin{array}{c}\text { Staphylococc } \\
\text { Es }\end{array}$ & $\begin{array}{c}\text { Acinetobacter } \\
\text { baumannii }\end{array}$ & $\begin{array}{c}\text { Staphylococcus } \\
\text { Epidermis }\end{array}$ & $\begin{array}{c}\text { Acinetoba } \\
\text { cter } \\
\text { baumannii }\end{array}$ & $\begin{array}{c}\text { Staphylococc } \\
\text { us } \\
\text { Epidermis }\end{array}$ & $\begin{array}{c}\text { Acinetobact } \\
\text { er }\end{array}$ \\
Baumannii \\
\hline 4 & - & - & - & - & - & - \\
2 & - & - & - & - & - & - \\
1 & - & - & - & - & - & - \\
0.5 & - & + & - & - & - & - \\
0.25 & - & + & - & - & - & - \\
0.125 & + & + & - & - & - & + \\
0.0625 & + & + & - & - & - & + \\
0.03125 & + & + & + & + & + & + \\
0.01562 & + & + & + & + & + & + \\
0.00781 & + & + & + & + & + & + \\
0.0039 & + & + & + & + & + & + \\
0.00195 & + & + & + & + & + \\
\hline
\end{tabular}

En effet, l'huile essentielle de Mentha piperita présente l'effet antibactérien le plus élevé avec des valeurs de CMI 8 fois et 6 fois moins importantes, en comparaison avec l'huile essentielle de M. pulegium contre $S$. epidermis et $A$. baumannii respectivement. En outre, il a été observé que A. baumannii (Gram négatif) était plus résistante aux huiles essentielles testées que $S$. epidermis (Gram positif). Cette résistance est étroitement liée à la composition de leur paroi cellulaire, car l'activité antibactérienne des huiles essentielles pourrait être expliquée par l'interaction moléculaire des groupements fonctionnels de leurs composants avec la paroi des bactéries ; ce qui provoque de profondes lésions. Cependant, la résistance des Gram négatif est attribuée aux contraintes de diffusion à travers leur membrane externe causée par la présence d'une barrière hydrophile (Trombetta et al., 2005). Nos résultats rejoignent les travaux de plusieurs recherches qui stipulent que les bactéries à gram positifs sont plus sensibles que les bactéries à gram négatifs (Amara et Boughérara, 2017 ; Ahmadi-Dastgerdi et al., 2017 ; Zhang et al., 2016).

\section{Conclusion}

L'enquête ethnobotanique a permis de préciser les différentes utilisations de Mentha pulegium, Mentha piperita et Pelargonium graveolens par la population de la ville de Taounate (Nord du Maroc) et de recueillir des informations sur la nature des usagers. Ce qui a révélé une multitude de résultats montrant que $41 \%$ des utilisateurs de ces plantes appartiennent à une tranche d'âge de 30 à 50 ans, $67 \%$ sont mariés, $56 \%$ sont des femmes et $32 \%$ 
sont analphabètes. Ce qui rassure sur la bonne transmission de la culture d'utilisation de ces plantes comme plantes médicinales.

Par ailleurs, le feuillage représente la partie la plus utilisée des plantes étudiés $(71 \%)$ et la décoction correspond au mode de préparation le plus pratiqué. Sur l'ensemble des maladies traitées, les affections digestives représentent les maladies les plus citées pour Mentha piperita (51\%), les affections respiratoires pour Mentha pulegium (50\%) et les affections dermatologiques pour Pelargonium graveolens (44\%). Les huiles essentielles des trois plantes étudiées ont montré une activité inhibitrice importante vis-àvis des souches microbiennes testées, avec des CMI variant de $0,01562 \%$ à $1 \%(\mathrm{v} / \mathrm{v})$.

Grâce aux vertus thérapeutiques précisées, et à la diversité de composition de leurs huiles essentielles, ces plantes pourraient être considérées comme source de molécules bioactives pour la formulation de nouveaux médicaments.

\section{References:}

1. Agnihotri VK., Agarwal SG., Dhar PL., Thappa RK., Baleshwara., Kapahi BK. (2005). Essential oil composition of Mentha pulegium L. growing wild in the North-western Himalayas India. Flavour Frag J 20: 607-10.

2. Ahmadi-Dastgerdi A., Ezzatpanah H., Asgary S., Dokhani S., Rahimi E. (2017). Phytochemical, Antioxidant and Antimicrobial Activity of the Essential Oil from Flowers and Leaves of Achillea millefolium subsp. millefolium. Journal of Essential Oil Bearing Plants 20: 395409.

3. Amara N., Boughérara Y. (2017). Activité Antimicrobienne de l'Huile Essentielle du Cyprès Vert (Cupressus sempervirens L.).Algerian Journal of Natural Products 5: 455-462.

4. Anyinam C. (1995). Ecology and ethnomedicine. Exploring links between current environmental crisis and indigenous medical practices. Soc Sci Medi 4:321-339.

5. Barros G., Magro A., Conceição C., Matos O., Barbosa A., Bastos MS. M., Mexia A. (2015). The use of Laurus nobilis and Mentha pulegium essential oils against Sitophilus zeamais (Coleoptera: Curculionidae) on stored maize. Rev Ciências Agrárias 38:191-5.

6. Benabed HK., Gourine N., Ouinten M., Bombarda I., Yousfi M. (2017). Chemical Composition, Antioxidant and Antimicrobial Activities of the Essential Oils of Three Algerian Lamiaceae Species. Current Nutrition \& Food Science 13: 97-109. 
7. Benchikha N., Djazi F., Lanez T. (2008). Chemical composition and in vitro antimicrobial activity of essential oils of Mentha piperita. $J$ Pharm Chem 2: 82-87.

8. Benkhnigue O., Zidane L., Fadli M., Elyacoubi H.,Rochdi A., Allal Douira (2011). Etude ethnobotanique des plantes médicinales dans la région de Mechraâ Bel Ksiri (Région du Gharb du Maroc). J Acta Bot Barc 53:191-216.

9. Boualem M., Mokhtar M., Saiah F., Benourad F., Bouhadiba R., Berkani A. (2017). Identification of Mentha piperita L. and Ricinus communis L. polyphenols by HPLC-DAD-ESI-MS and evaluation of their insecticidal properties against Aphis spiraecola P. South Asian Journal of Experimental Biology 7: 28-34.

10. Bouari C, Bolfa P, Borza G, Nada`s G, Ca toi C \& Fit N (2014) Antimicrobial activity of Mentha piperita and Saturenja hortensis in a murine model of Cutaneous protothecosis. Journal de Mycologie Médicale 24: 34-43.

11. Boukhatem MN., Kameli A., Saidi F. (2013). Essential oil of Algerian rose-scented geranium (Pelargonium graveolens): Chemical composition and antimicrobial activity against food spoilage pathogens. Food Control 34:208-213.

12. Boukhris M., Ben Nasri-AyachiM., Mezghani I., Bouaziz M., Boukhris M., Sayadi S (2013). Trichomes morphology, structure and essential oils of Pelargonium graveolens L (Geraniaceae). Ind Crops Prod 50:604-610.

13. Bouyahya A., Et-Touys A., Bakri Y., Talbaui A., Fellah H., Abrini J., Dakka N. (2017). Chemical composition of Mentha pulegium and Rosmarinus officinalis essential oils and their antileishmanial, antibacterial and antioxidant activities. Microbial pathogenesis 111: 41-49.

14. Chraibi M., Farah A., Lebrazi S., El Amine O., Iraqui Houssaini M., Fikri-Benbrahim K. (2016). Antimycobacterial natural products from Moroccan medicinal plants: Chemical composition, bacteriostatic and bactericidal profile of Thymus satureioides and Mentha pulegium essential oils. Asian Pacific Journal of Tropical Biomedicine 6: 836840.

15. Chraibi M., Benbrahim FK., Elmsellem H., Farah A., Abdel-Rahman I., El Mahi B.,Hlimi F. (2017). Antibacterial activity and corrosion inhibition of mild steel in $1.0 \mathrm{M}$ hydrochloric acid solution by $M$. piperita and $M$. pulegium essential oils. Journal of Materials and Environmental Sciences 8: 972-981.

16. Chraibi M., Fikri-Benbrahim K., Ou-Yahia D., Farah A. (2017). African peppermint (Mentha piperita) from Morocco: Chemical 
composition and antimicrobial properties of essential oil. $J A d v$ Pharma Tech Research $8: 86-90$.

17. Desam NR., Al-Rajab AJ., Sharma M., Mylabathula MM., Gowkanapalli RR., Albratty M. (2017). Chemical constituents, in vitro antibacterial and antifungal activity of Mentha piperita L. (peppermint) essential oils. Journal of King Saud UniversityScienceArticle in press.

18. El Rhaffari L., Zaid A. (2002). Pratique de la phytothérapie dans le sud-est du Maroc (Tafilalet): Un savoir empirique pour une pharmacopée rénovée. Horizon 293-318.

19. Franchomme P., Pénoël D., Jollois R. (1990). L'aromathérapie exactement- Encyclopédie de 1'utilisation thérapeutique des huiles essentielles, Fondements, démonstration, illustration et applications d'une science médicale naturelle. Editions Jollois 445p.

20. Hafsé M., K. Fikri-Benbrahim, A. Farah (2015). Ethnobotanical survey on the use of Pistacia lentiscus in northern MOROCCO (Taounate). Int J Innov Appl Studies 13 (4): 864-872.

21. Hamidpour R., Hamidpour S., Hamidpour M., Marshall V., HamidpourR. (2017). Pelargonium graveolens (Rose Geranium)-A Novel Therapeutic Agent for Antibacterial, Antioxidant, Antifungal and Diabetics. Archives in Cancer Research.5 (1): 134.

22. Kogiannou DA., Kalogeropoulos N., Kefalas P., Polissiou MG., Kaliora A. (2013). Herbal infusions; their phenolic profile, antioxidant and anti-inflammatory effects in HT29 and PC3 cells. Food Chem Toxicol 61: 152-159.

23. Labarde S. (2017). La trousse à pharmacie aromathérapique du sportif. Actualités Pharmaceutiques 56: 42-46.

24. Lakhdar L., Farah A., Bajjou T., Rida S. (2015). In vitro Antibacterial Activity of Essentials Oils from Mentha pulegium, Citrus aurantium and Cymbopogon citratus on Virulent Strains of Aggregatibacter actinomycetemcomitans. Int $J$ Pharmacognosy phytochemical Res 6:1035-1042.

25. Lamendin H. (2004). Huiles essentielles en diffusion atmosphérique. Chir. Dent. Fr1185 : 78-80.

26. Li Y., Liu Y., Ma A., Bao Y., Wang M., Sun Z. (2017). In vitro antiviral, anti-inflammatory, and antioxidant activities of the ethanol extract of Mentha piperita L. Food Science and Biotechnology 26: 1675-1683.

27. Lorrain E. (2013). 100 questions sur la phytothérapie. Ed. La boétie, Italie.

28. Mogosan C., Vostinaru O., Oprean R., Heghes C., Filip L., Balica G., Moldovan RI. (2017). A Comparative analysis of the chemical 
composition, anti-inflammatory, and antinociceptive effects of the essential oils from three species of Mentha cultivated in Romania. Molecules 22: 263.

29. Parsania M., Rezaee MB., Hamidreza Monavari S., Jaimand K., Milad Mousavi-Jazayeri S., Razazian M., Nadjarha MH. (2017). Antiviral screening of four plant extracts against acyclovir resistant herpes simplex virus type-1. Pakistan journal of pharmaceutical sciences 30(4): 1407-1411.

30. Radan C. (2017). Dermatite atopique et psoriasis, deux pathologies cutanées auto-immunes. Actualités Pharmaceutiques 56 : 44-47.

31. Sachin J., Dinesh Kumar J., Neelam B. (2012). In-Vivo Antioxidant activity of ethanolic extract of Mentha pulegium leaf against $\mathrm{CCl} 4$ induced toxicity in rats. Asian Pacific Journal of Tropical Biomedicine 737-740.

32. Saraswathi J., Venkatesh K., Nirmala B., MajidHameed H., Roja Rani A. (2011). Phytopharmacological importance of Pelargonium species. Journal of Medicinal Plants Research 5(13): 2587-2598.

33. Teixeira B., Marques A., Ramos R., Batista I., Serrano C., Matos O. (2012). European pennyroyal (Mentha pulegium) from Portugal: chemical composition of essential oil and antioxidant and antimicrobial properties of extracts and essential oil. Ind Crop Prod 36: $81-7$.

34. Trombetta D., Castelli F., Sarpietro MG., VenutiV., Cristani M., Daniele C., Antonella S., Gabriela M., Giuseppe B., Maria G. (2005). Mechanisms of antibacterial action of three monoterpenes. $J$ Antimicrob Agents Chemother 49 :2474-2478.

35. Zhang Y., Liu X., Wang Y., Jiang P., Quek S. (2016). Antibacterial activity and mechanism of cinnamon essential oil against Escherichia coli and Staphylococcus aureus. Food Control 59: 282-289. 